\title{
REVIEWS
}

\section{Communication in bacteria: an ecological and evolutionary perspective}

\section{Laurent Keller* and Michael G. Surette}

\begin{abstract}
Individual bacteria can alter their behaviour through chemical interactions between organisms in microbial communities - this is generally referred to as quorum sensing. Frequently, these interactions are interpreted in terms of communication to mediate coordinated, multicellular behaviour. We show that the nature of interactions through quorum-sensing chemicals does not simply involve cooperative signals, but entails other interactions such as cues and chemical manipulations. These signals might have a role in conflicts within and between species. The nature of the chemical interaction is important to take into account when studying why and how bacteria react to the chemical substances that are produced by other bacteria.
\end{abstract}

Secondary metabolite Chemical produced by the cell that is not essential for maintenance of cellular function or for normal growth of the organism.

Quorum sensing A system by which bacteria communicate and obtain information on bacterial density in their environment.

* Department of Ecology and Evolution, University of Lausanne, 1015 Lausanne, Switzerland.

${ }^{\ddagger}$ Department of Microbiology and Infectious Diseases, Department of Biochemistry and Molecular Biology, University of Calgary, 3330 Hospital Drive NW, Calgary, Alberta T2N 4N1, Canada. e-mails: laurent.keller@unil. ch;surette@ucalgary.ca doi:10.1038/nrmicro1383 Published online

27 February 2006
Over the last two decades, our view of bacteria has dramatically changed. Bacteria have often been studied as populations of cells that act independently, but it now seems that there is much interaction and communication between cells. Bacteria can produce an extensive repertoire of secondary metabolites, and can respond to a wide variety of chemicals in their environment. In recent years, particular groups of secondary metabolites have been characterized for their role in the regulation of gene expression in a cell-density-dependent manner, and this behaviour has been collectively referred to as quorum sensing, or cell-cell communication (FIG. 1). In its simplest form, this process results from the production and accumulation of signalling molecules in the surrounding environment. The signalling molecules, also referred to as autoinducers, bind to receptors on, or in, the bacterial cell, which leads to changes in gene expression at some threshold concentration. Quorum sensing is generally thought to act as a mechanism for the coordinated regulation of behaviour at the level of populations of cells.

The realization that bacteria can communicate, cooperate and alter their behaviour, according to changes in their social environment, has led to an explosion of research in this area, exemplified by an Entrez PubMed search for 'quorum sensing' that resulted in more than 900 articles and 150 reviews in the last 10 years (for recent reviews see REFS 1-5). Most studies have focused on the molecular aspects of cell-cell communication, and much less attention has been paid to the ecological context of why bacteria produce signalling molecules and respond to both intraspecific and interspecific signals ${ }^{6}$. One view among microbiologists is that quorum sensing simply evolved because it allows bacteria to coordinate the behaviour of the group and take on some of the characteristics of multicellular organisms. However, large bodies of theoretical work and empirical studies in other organisms have shown that cooperation and communication can evolve only under very restricted conditions $\mathrm{s}^{7-13}$. Moreover, the role of quorum-sensing signals in cell-cell communication has been challenged. It has been suggested that autoinducers function as probes to provide individual cells with information about the diffusive and mixing properties of the immediate environment ${ }^{14}$. Whereas this might be true in some cases (for example, in the Staphylococcus aureus agr system ${ }^{15}$ ), it is at odds with the biology of some well-studied systems (for example, Vibrio fischeri in the bobtail squid ${ }^{16-19}$ ). In any case, this raises the important issue that signalling pathways might sometimes have evolved for reasons other than cell-cell signalling, or might have acquired new functions once they were established.

The aim of this review is to discuss the general issue of quorum sensing and cell-cell communication from an evolutionary and ecological perspective. Over the last four decades, there have been extensive studies on the evolution of communication and cooperation in vertebrates, insects and other social animals, leading to the founding of a solid theoretical framework ${ }^{20}$. Using this framework, we discuss why it is important to consider not only the opportunity for cooperation, but also 


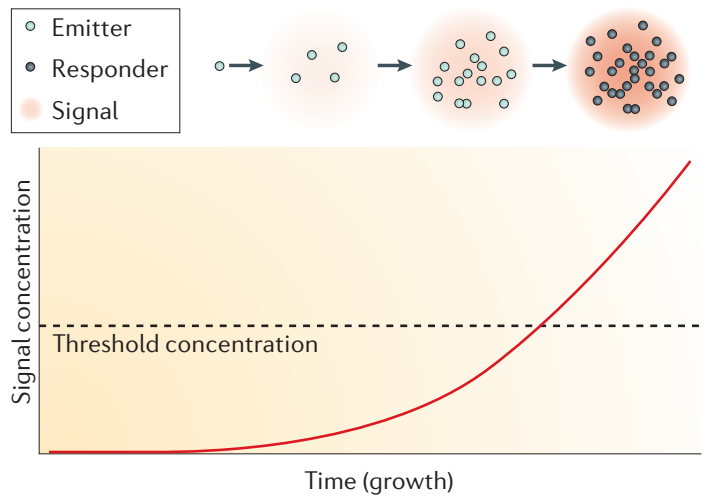

Figure 1 | Generic scheme for quorum sensing. In its simplest form, cell-cell signalling results from the production of signalling molecules by emitter cells and their accumulation in the surrounding environment. At some threshold concentration, the signalling molecules bind to receptors on or in the bacterial cell, leading to changes in gene expression in the responding cell. For intraspecies quorum sensing, the emitter and responder are usually the same cells, as illustrated here. Often, but not always, the genes that are involved in synthesis and response activate their own expression - explaining the term autoinducer. A signalling molecule is considered to act at low concentrations and not to be involved in primary metabolism.

Autoinducer

A system by which bacteria communicate. Signalling molecules are chemicals, similar to pheromones that are produced by an individual bacterium, which can affect the behaviour of surrounding bacteria.

Signal

Any act, structure or chemical emission that alters the behaviour and gene expression of other organisms which evolved because of that effect, and that is effective because the receiver's response has also evolved.

$\mathrm{N}$-acyl homoserine lactone A group of intercellular signalling molecules, produced by some Gram-negative bacteria, made up of a homoserinelactone ring with an $\mathrm{N}$-linked acyl side chain

LuxS/autoinducer-2 (Al-2). Refers to a conserved gene (luxS) found in both Gram-negative and Grampositive bacteria that mediates production of a common signalling molecule autoinducer-2.

Pre-protein

A protein initially synthesized as an inactive form that undergoes proteolytic cleavage to release the active protein or peptide competition between individuals living in the same environment. Throughout the review, we attempt to apply the established terminology in the field of ecology and evolution to bacterial cell-cell signalling.

\section{Cost and specificity of cell-cell signalling}

It is currently unclear how commonly cell-cell signalling occurs in bacteria. A survey of the distribution of $\mathrm{N}$-acyl homoserine lactone (AHL)-based signalling within bacteria challenged the widespread view that quorum sensing is very common ${ }^{21}$. Nonetheless, the types of potential chemical molecules that bacteria produce and respond to in order to adapt to their environment, including competitive environments with other microorganisms, remain largely underexplored, and the repertoire of chemical molecules that are associated with cell-cell signalling continues to grow. Currently, there are three well-defined classes of molecules that serve as the paradigms for chemical signalling in bacteria: oligopeptides, AHLs and the LuxS/autoinducer-2 (AI-2) class. These three systems will be considered from an evolutionary perspective, in particular in terms of two key components of communication: the cost associated with signalling and information specificity.

It is difficult to precisely define the costs of communication in bacteria. The quantity of the signal produced and the level required to elicit a response are not well defined, particularly in natural environments. Furthermore, this varies not only for each of the three systems, but within each system as well. The protein machinery for the production and export of the signals acts catalytically, and can be assumed to be roughly equivalent in each system. Therefore, we consider that the costs are primarily the metabolic burden that is associated with signal production. The three signalproduction biochemical pathways and their associated metabolic costs are presented in FIG. 2 and TABLE 1, respectively. Costs are also related to the quantity of signal produced, though the relationship between amount and cost will vary for each signalling pathway. Based on the concentration of signal required for a response, it can generally be stated that the amounts produced are inversely proportional to the production costs. An additional consideration in signal production is the specificity of the signal produced. This is in part a measure of the information content of the signal, and specificity generally correlates with the cost of production.

The first of the cell-cell-signalling mechanisms is oligopeptide signalling, which is the predominant signal used by Gram-positive bacteria (FIG. 2a). Typically, a preprotein is generated, processed into the active signalling peptide and exported from the cell ${ }^{4,22}$. The biochemical cost of synthesis is relatively expensive, even for short peptides (TABLE 1). The chemical structure of the signal is precisely defined by the sequence of amino acids, which might be further modified, such as the formation of a thiolactone ring in the $S$. aureus signalling peptides ${ }^{23}$. The oligopeptide signals are highly specific, sometimes allowing distinct signalling within different strains of the same species. This specificity is exemplified by $S$. aureus strains, which are classified according to their oligopeptide signals ${ }^{24,25}$. Groups are defined as a collection of strains that produce signalling peptides that can cross-activate. Equally significant, the signalling peptides within a particular group act as potent inhibitors of signalling in other groups. In S. aureus, and probably other species, it therefore seems that the signalling is highly specific and has a role in intraspecific competition, as well as in coordinated, cooperative behaviour.

The second established paradigm in cell-cell signalling is through AHLs (FIG. 2b). In Gram-negative bacteria, the production of AHLs involves the reaction of $S$-adenosylmethionine (SAM) with an acyl-acyl carrier protein (acyl-ACP), which is typically carried out by an enzyme of the LuxI family ${ }^{1,26,27}$. Acyl-ACP molecules are intermediates in fatty-acid biosynthesis. Both these substrates have an associated metabolic burden for synthesis, and there is an intermediate cost associated with production (TABLE 1). The specificity of this system is only moderate, in that a typical LuxI protein will make one predominant AHL, and one or more minor AHLs. For example, the Pseudomonas aeruginosa AHL synthase LasI makes both 3-oxodoecanol-homoserine lactone and 3-oxohexnoyl-homoserine lactone ${ }^{28,29}$ (FIG. 2b). The AHL signal is typically detected by a member of the LuxR family of transcriptional regulators. The receivers also show some relaxed specificity, as different LuxR proteins differ in the AHL molecules that they recognize, as well as in the number of variants that they can detect. In some cases, the LuxR protein will recognize a wide range of AHLs, such as the Agrobacterium tumefaciens and Chromobacterium violaceum proteins, which have been exploited to generate general sensors for a wide range of AHL molecules ${ }^{29,30}$. 
a

Preprotein

ñ

(N)
Oligopeptide

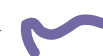

\section{b}
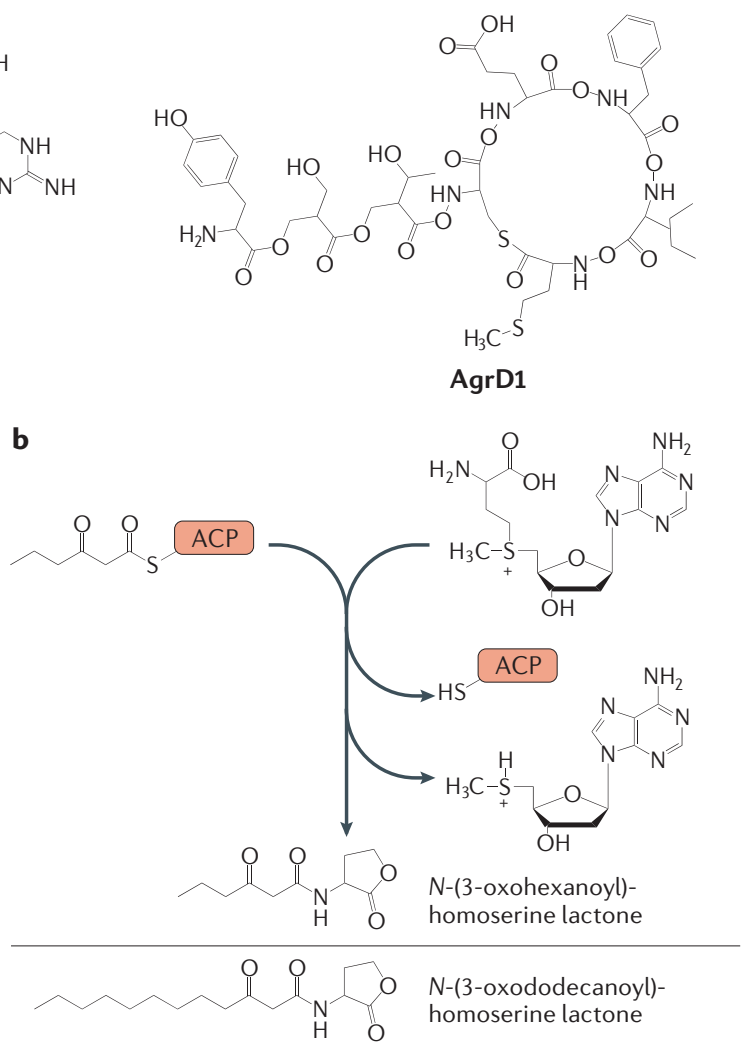

c

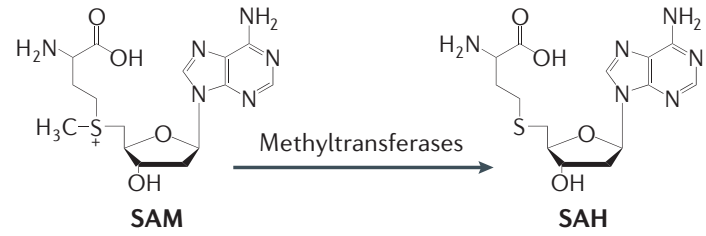

Adenine

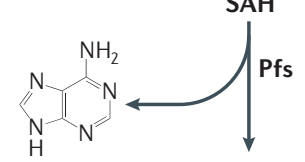

Hcy<smiles>NC(CCS)C(=O)O</smiles>
$\mathrm{H}_{2}$ 年 
Chemical manipulation

Chemical emission that alters the behaviour and gene expression of other organisms. However, contrary to a signal, the effect induced by chemical manipulation has a negative effect on the fitness of the receiver.

Cue

An act, structure or chemical emission that alters the behaviour and gene expression of other organisms. However, contrary to a signal, it did not evolve specifically for that effect. from cheating by not producing the signal ${ }^{40,41}$. To illustrate this point, imagine a colony of bacteria in which one cell stops producing the signal. This would remove the burden that is associated with signal production, yet allow this cell to benefit from the coordinated behaviour within the colony. Therefore, signal production might frequently be an altruistic behaviour that provides benefits to the colony, but is associated with a cost for the individual producing the signal. Similarly, the response to a signal might incur a cost to the individual, but benefit the colony. The question, therefore, becomes why do bacteria produce costly signals or respond to such signals if it increases the fitness of other individuals at a personal cost? After all, the principle of natural selection is to favour those individuals that have higher survival and fecundity than their neighbours. To address this question, we first need to outline the possible modes of communication between organisms in relation to the consequences on the fitness of the emitter and receiver.

\section{Signals, cues and chemical manipulation}

Communication has evolved in many organisms, with the most sophisticated forms being found in social organisms, in particular humans. For true communication to occur, two conditions must be met. First, one or several individuals must produce a signal that can be perceived by other individuals, and second, the perceivers must alter their behaviour in response to this signal (BOX 1). Importantly, communication can be selected for and remain stable over evolutionary time only if both the emitter and the receiver gain benefits from communicating ${ }^{42}$. Because producing a signal implies a cost, natural selection will select against signal production if the change of behaviour in the receiver does not translate into a benefit for the emitter. Inversely, a receiver's responses to a chemical, acoustic or visual signal will be selected against if it does not translate into a direct benefit and increase in individual fitness. In other words, communication between two parties will evolve and remain stable only if both parties benefit from the transfer of information that is conveyed by the signal.

It is important to realize that the mere demonstration of bacteria responding to a chemical substance produced by other bacteria does not necessarily imply communication. In general, there are two situations in which changes in behaviour can occur without communication: when bacteria use information from chemicals that are produced for purposes other than communication, or when there is chemical manipulation by other bacteria living in the same environment. In the first situation, imagine that bacterial species A benefits when it obtains information on the density of species B in the environment. In such cases, individuals of species A will be selected to use any reliable cue that provides information on the density of species B (BOX 1). This could, for example, be a metabolic residue that is produced by $B$. In this case, one cannot say that species A and B communicate. Rather, the metabolite that is produced by B is used as an informational cue by A to adaptively change its own behaviour, perhaps at the detriment of individuals of species B. A variation on this is eavesdropping, in which the metabolite that is sensed by $A$ is an autoinducer that is produced by B. In this case, the same autoinducer substance functions as a signal for communication between cells of species B, and as a cue for members of species $A$ to access information on species B's density.

An example of an autoinducer signal of one species being used as a cue by another might be the interaction of oropharyngeal flora with the opportunistic pathogen P. aeruginosa in cystic fibrosis. In this case, the Grampositive oropharyngeal flora produces significant levels of AI-2, whereas P. aeruginosa does not produce AI-2 but does respond to it ${ }^{43}$. In this interaction, there is probably no benefit for the Gram-positive flora to associate with, or provide information to, $P$. aeruginosa. Accordingly, it is unlikely that AI-2 is produced by the Gram-positive flora as a means to communicate with $P$. aeruginosa. AI-2 is therefore best described as a cue that is used by P. aeruginosa, rather than a signal that evolved for interspecific communication.

The second situation in which behavioural change without communication can occur is if there is chemical manipulation (BOX 1). There are increasing examples of parasites that manipulate the behaviour of their host by producing chemical substances that interfere with the host's neuroendocrine system ${ }^{44-46}$. In the same vein, it is conceivable that some bacteria might chemically manipulate other bacteria to behave against their own interests. In that case, the action of the chemical substance should not be regarded as communication, because one of the

\section{Table 1 | Metabolic costs of signal production in bacterial cell-cell-signalling systems}

\begin{tabular}{|c|c|c|}
\hline Signal & Metabolic Cost* & Example \\
\hline Autoinducer-2 & Low/none & 0-1 ATP \\
\hline
\end{tabular}




\section{Box 1 | Terminology used to describe chemical interactions}

\section{Signal}

Any act, structure or chemical emission that alters the behaviour and gene expression of other organisms, evolved because of that effect and is effective because the receiver's response has also evolved.

\section{Cues}

Also an act, structure or chemical emission that alters the behaviour and gene expression of other organisms. However, contrary to a signal, it did not evolve specifically for that effect.

\section{Chemical manipulation}

Chemical emission that alters the behaviour and gene expression of other organisms. However, contrary to a signal, the effect induced by chemical manipulation has a negative effect on the fitness of the receiver.

partners would benefit by not responding to the chemical substances that are produced by the other species.

A possible example of chemical manipulation comes from interactions in complex communities that form dental plaque in the human oral cavity. These communities form temporal and spatial arrangements of different bacteria ${ }^{3,47,48}$. Although the complexity of interactions in these communities is only beginning to be unravelled, interactions between species have been characterized in a few instances, such as for Streptococcus gordonii and Veillonella atypica, two species that are involved in the early colonization of the dental enamel. V. atypica requires $S$. gordonii to be present to colonize dental surfaces because $S$. gordonii ferments sugars and releases lactic acid, which is the preferred carbon source for $V$. atypica. Interestingly, it has been shown that $V$. atypica produces a soluble chemical that induces amylase expression in S. gordonii, thereby increasing the degradation of complex carbohydrates and lactic-acid production ${ }^{49}$. As there is apparently no direct benefit for S. gordonii to respond to the substance that is produced by V. atypica, this indicates that this might be a case of chemical manipulation whereby the emitter alters the behaviour of the receiver to its own benefit.

It is important to differentiate between signals, cues and chemical manipulation, which differentially affect the fitness of the emitter and the receiver. For example, receivers will generally be selected for greater sensitivity to chemical signals; receivers better able to resist chemical manipulation, however, will be favoured over evolutionary time. Therefore, pathways that are involved in chemical manipulation and communication through chemical signals should evolve differently over time.

Elucidating the effects of chemicals on the fitness of emitters and receivers might have some medical implications. In the case of the interaction of V. atypica and S. gordonii on tooth enamel, it would probably be more difficult to interfere with the cellular interactions if it was a case of chemical manipulation rather than true communication. This is because, with chemical manipulation, the emitter is selected to produce chemicals that elicit a response to which the receiver cannot escape. In other words, under chemical manipulation, there has been a long evolutionary arms race between the emitter and the receiver, with the effect being that the chemi- cals that are currently used are highly efficient, possibly directly acting on the physiology of the responder. By contrast, in a situation in which there is no conflict, the receiver has not been selected to escape the effect of the chemicals that are produced by the emitter and, accordingly, such systems are likely to be less resilient to external perturbations.

\section{Conditions for the evolution of communication}

Because communication incurs a cost in terms of signal production, it is important to understand the conditions under which a behaviour that favours others at individual expense can evolve. Contrary to the expectation that is derived from natural selection, among many social species some individuals behave altruistically and forego some of their direct reproduction to help other individuals. In the most extreme cases, the physiology of some individuals (for example, ant workers) precludes reproduction entirely. How can characteristics that prevent individuals from transmitting copies of their own genes to descendants arise and persist?

Intraspecific interactions between bacteria. In species that lack complex cognitive abilities, the only possible selective force that promotes cooperation during intraspecific interactions is kin selection, as elegantly demonstrated more than 40 years ago by the evolutionary biologist William D. Hamilton ${ }^{8,9}$. The general principle of kin selection is that altruistic acts that are directed toward relatives produce an important kind of reproductive compensation (BOX 2). By enhancing the reproduction of relatives, a help-inducing gene indirectly propagates copies of itself in those relatives. The power of Hamilton's theory was that it showed that help-inducing genes benefit in an essentially identical way when the help is directed towards non-descendant relatives, such as siblings.

Kin-selection theory gives the conditions under which an altruistic act will be positively selected. It involves three terms: the change in the actor's personal fitness, the change in the recipient's personal fitness, and the relatedness (meaning the proportion of genes shared by common ancestry) between the actor and the recipient. A general description of Hamilton's rule is that altruistic acts are more likely to be selected for when individuals are closely related and when the decrease in the actor's personal fitness is relatively small compared to the increase in the recipient's fitness. In bacteria, therefore, the three factors that are conducive to intraspecific communication are high relatedness between bacteria (which is the case for clonal groups that are derived from a single founding cell; see FIG. 3), low cost of signal production (for example, AI-2-dependent signalling) and high benefit for bacteria to behave in a coordinated manner (for example, coordinate regulation of virulence genes or in fruiting body formation in Myxococcus).

Inversely, communication should be less common when multiple strains of the same species of bacteria are mixed, when signal production is costly or when the benefits of coordinated behaviour are limited. An experimental study with the pathogenic bacterium $P$. aeruginosa showed that higher relatedness results in higher levels of 


\section{Cheater}

An individual obtaining benefits from a collectively produced public good that are disproportionally large relative to its own contribution to that good.

Conjugative plasmid A plasmid that can move from one cell to another during the process of conjugation. cooperation, as demonstrated by a higher investment in the production of siderophore iron-scavenging agents in lines that are kept under higher relatedness ${ }^{50}$. Similarly, experiments in which strains of Myxococcus xanthus were competed against one another in all possible pairwise combinations showed that in most pairings, at least one competitor showed strong antagonism towards its partner ${ }^{51}$. These data show that bacteria can perceive the presence of different strains and that changes in overall relatedness might have profound effects on population growth and survival.

One group of bacteria in which signalling seems to be particularly prevalent is in bacterial pathogens (although the view that signalling is more prevalent in these bacteria might be a result of bias from an emphasis on research on pathogens). Importantly, pathogens might frequently form microcolonies with clonal individuals when there is local colonization within the host (FIG. 3), therefore providing the prerequisites for signalling to evolve. In this situation, coordinated expression of virulence determinants would be most advantageous when the cell number in the microcolony reaches a critical threshold.

Similarly, bacteria in the environment might use quorum sensing to regulate expression of extracellular enzymes that degrade macromolecules. As both the enzymes and their products will diffuse away from the cell or cells, there is an advantage to initiating catabolic production when there are sufficient numbers of cells to scavenge all the products. This is clearly more efficient in a microcolony than in single isolated cells, and coordinate expression of these enzymes using intraspecies signalling systems would be optimal. In this respect, it is interesting to note that many of the virulence determinants that are regulated through quorum-sensing pathways are extracellular.

\section{Box 2 | Kin selection}

Hamilton's rule spells out kin-selection theory by giving the conditions under which an altruistic act will be positively selected. It involves three terms: the change in the actor's personal fitness, the change in the recipient's personal fitness, and the relatedness between the actor and the recipient. The degree of relatedness is a measure of the genetic similarity between these two individuals, and is equal to the probability that a random gene of the recipient has an identical copy, by descent, in the actor. So, a general description of Hamilton's rule is that altruistic acts are more likely to be selected for when individuals are closely related and when the decrease in the actor's personal fitness is relatively small compared to the increase in the recipient's fitness.

An individual helping a relative indirectly promotes the transmission of copies of its own genes to the next generation. How many of its genes will be transmitted depends on the relatedness between both individuals, the benefit that the altruistic act brings to the recipient, and the induced cost for the altruistic individual. Benefits and costs typically represent differences in the number of descendants, which is the basic unit used in evolutionary biology. If the degree of relatedness between an actor and a recipient is $r$, the cost to the altruistic individual is $c$ and the benefit for the beneficiary is $b$, the altruistic act will be favoured when $(b \times r)-c>0$.

Here is a simple example to illustrate Hamilton's rule. Imagine a gene that programmes an individual to die so as to save relatives' lives. One copy of the gene will be lost if the altruist dies, but the gene will increase in frequency in the population if, on average, the altruistic act saves the lives of more than 2 siblings $(r=0.5)$, more than 4 nephews or nieces $(r=0.25)$, or more than 8 cousins $(r=0.125)$. J.B.S. Haldane fully apprehended kin-selection theory and Hamilton's rule when he announced, having done some calculations on an envelope in a pub, that he would be ready to give his life to save 2 brothers or 8 cousins!
In the simplest case of bacterial colonization, microcolonies are probably established from single cells, as in infections in which bacteria establish on mucosal surfaces (some pathogens), in the environment in which they might colonize new sites or even in liquid environments that are not well-mixed. In these instances, cellcell-signalling control of gene expression might provide a selective advantage to the wild-type over the signalling-deficient strains (FIG. 3). This would be the case in which cell-cell signalling controls extracellular enzymes and/or virulence factors. Even if signalling-deficient mutant strains (cheaters) accumulated over time in the community, they would be at a selective disadvantage in the next colonization event and would therefore not have an advantage over time. This assumes that colonization is initiated clonally by a single cell and that (at least initially) cell-cell signalling operates within the microcolony and not between microcolonies ${ }^{40}$.

There are examples, however, of signalling within groups of non-clonal bacteria, such as $M$. xanthus. In these organisms, individual cells aggregate under starvation conditions and form fruiting bodies (FIG. 3). Within these fruiting bodies, some cells develop into spores and the others $\mathrm{die}^{52-56}$. In M. xanthus, this process is mediated by two signalling pathways: the first (A-signalling) leads to aggregation of cells, and the second (C-signalling) involves the formation of a mound and, ultimately, fruiting-body formation ${ }^{54,56,57}$. The $\mathrm{C}$-signalling requires cell-cell contact, as the signalling molecule is on the surface of the cell ${ }^{58,59}$. Cheaters that are defective in either A- or C-signalling have been identified which are overrepresented in the spore-forming population when grown in mixed colonies with wild-type cells. When grown in monoculture, they produce spores at a lower frequency than wild-type cells ${ }^{60,61}$. If one considers the wild-type behaviour to be altruistic, then these cheaters take advantage of wild-type cells, but fare less well on their own.

Another exception to the notion that intraspecies cell-cell signalling occurs predominantly within clonal groups is the role of peptide signalling in conjugative plasmid transfer in Enterococcus species ${ }^{62,63}$. In a population of cells that all contain plasmid, inhibitory signals prevent non-productive mating from occurring. The signalling oligopeptide produced in cells that lack the conjugative plasmid is detected by donor cells that contain the plasmid. Initially, cells produce an aggregation substance that promotes the physical association of cells to facilitate efficient conjugation-mediated plasmid transfer. This system necessarily functions between non-clonal cells, and allows for the very efficient transfer of these conjugal plasmids in mixed populations of Enterococcus species. Of clinical concern is the fact that antibioticresistance genes, in particular those encoding resistance to vancomycin, reside on these plasmids.

Interspecific interactions between bacteria. Cooperation between species also poses a difficult challenge to evolutionary theory because conditions under which individuals pay a cost to benefit another species are rare ${ }^{20}$. However, two main conditions that might be conducive to interspecific cooperation are partner fidelity and part- 
a

3

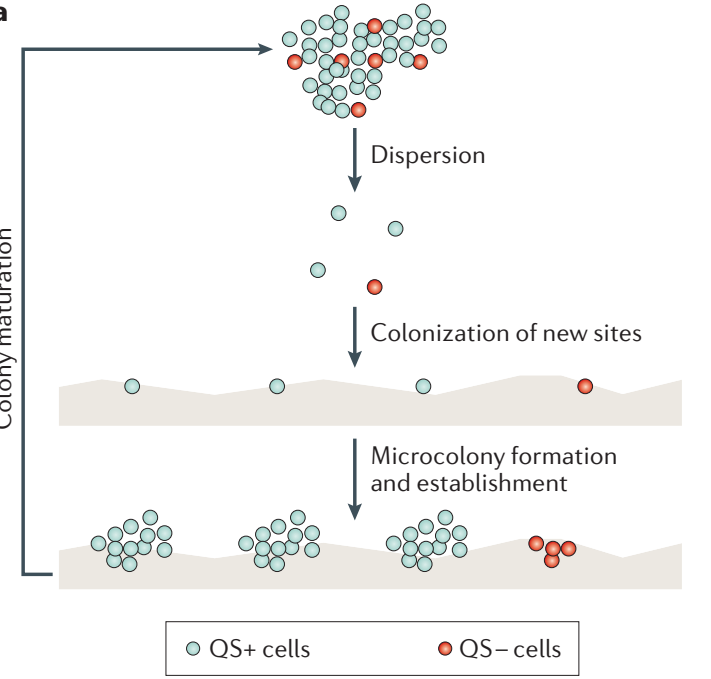

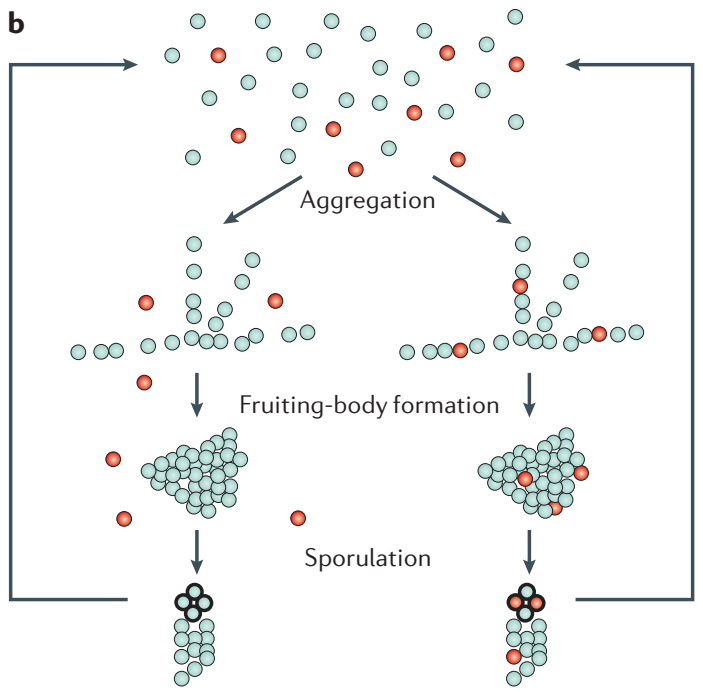

Figure 3 | Clonal relationship and intraspecies signalling. a | For most quorum-sensing bacteria, intraspecies cell-cell signalling predominates in clonal populations of cells. The role of cell-cell signalling is hypothesized to function most significantly early during colonization of a new site. Microcolonies will form from single cells and will therefore be clonal in nature. Where cell-cell signalling would provide a competitive advantage, quorum-sensing-positive (QS+) cells will form microcolonies more efficiently. Even if cheaters accumulate within a maturing community, these cells will be at a disadvantage in subsequent colonization events. For example, in Pseudomonas aeruginosa it has been shown that quorum sensing has an important role in colonization and virulence in cystic fibrosis, and in most patients it seems that infection is established clonally ${ }^{91}$. Isolates from patients are predominantly $\mathrm{N}$-acyl homoserine lactone (AHL) producers, however isolates that are defective in $\mathrm{AHL}$ production are also found ${ }^{92}$. This behaviour is not restricted to adhered populations, as illustrated, but could occur effectively in non-attached populations of aggregates or cells that grow in static conditions in which local concentrations of signals (and extracellular enzymes) could accumulate around cells. $\mathbf{b} \mid$ For bacteria that undergo fruiting-body formation and sporulation, such as Myxococcus species ${ }^{52-56}$, colony formation is fundamentally different. The initial event involves the aggregation of cells (triggered by a signalling pathway called A-signalling, in the case of Myxococcus xanthus) followed by the formation of a fruiting body (involving a contact-dependent signalling pathway called C-signalling). Last, a fraction of cells within the fruiting body develop into spores (thick black outline) and will seed the next generation of free-living cells. Because the initial event is an aggregation of cells within a local area, there is no guarantee of clonality. The pathway on the left would prevail if there was a mechanism to prevent non-signalling cells from aggregating, and the pathway on the right would occur if cheating occurs.

ner choice (for an example, see REF. 20). Partner fidelity describes a situation in which two or more individuals of different species are associated for an extended series of exchanges. Under such conditions, the fitness of each partner is positively dependent on the fitness of the other. Biological examples that fulfil this condition include vertically transmitted symbionts and commensals (for example, mitochondria and ant-acacia symbiosis).

In bacteria, it is unclear whether such situations are frequently met. A possible example would be syntrophic interactions - for example, in the case in which complete degradation of aromatic compounds by fermentative pathways becomes energetically favourable only if the bacteria that carry out the first biochemical steps are associated with methanogens or sulphur-reducing bacteria that further metabolize the products ${ }^{64}$. However, it is as yet unclear whether there is partner fidelity in such syntrophic associations and whether species have co-evolved to establish efficient systems of interspecific communication and cooperation, or whether species simply preferentially associate with others having complementary biochemical machinery.

Partner choice, the other main condition that allows for interspecific mutualisms, occurs when individuals interact and reward other cooperative individuals, but avoid rewarding less cooperative individuals. Choice might take several forms, ranging from establishing cooperation with only one of several potential partners to altering the duration of cooperation with a partner according to its own propensity to cooperate. It is unclear to what extent partner choice does occur in bacteria and, if so, under what conditions. In some respects, a possible example would be the plasmid-mating systems of Enterococcus species, in which signalling operates to avoid costly unproductive matings between only recipient cells or only donor cells ${ }^{62,63}$.

Although there are many examples showing that bacteria respond to chemical substances that are produced by other organisms, there are no conclusive examples of communication systems that have specifically evolved for interspecific interactions. For example, interspecies interactions, such as crosstalk between AHL signals between two cystic-fibrosis pathogens, $P$. aeruginosa and Burkholderia cepacia ${ }^{65,66}$, probably do not represent an evolved, coordinated behaviour, but rather a fortuitous overlap in signal molecules that are produced and the range of AHLs to which each bacteria can respond. Indeed, the clinical association of these two bacteria is 
a

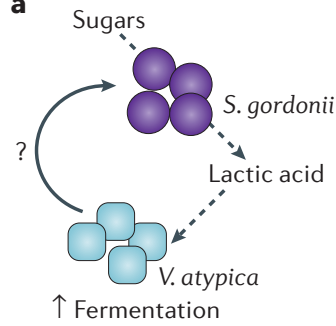

b

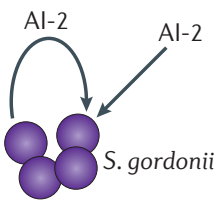

$\uparrow$ Biofilm formation

$\uparrow$ Sugar metabolism c

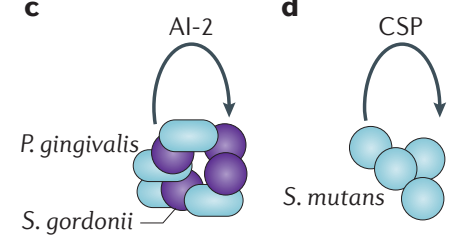

$\uparrow$ Mixed biofilm formation d

$\uparrow$ Competence

Figure 4 | Chemical signalling within microbial communities of oral bacteria. Biofilm communities within the oral cavity are characterized by complex spatial and temporal organization. Many examples of chemical signalling within these communities have been described. Some examples are presented here. a| Veillonella atypica produces a soluble chemical that induces amylase expression in Streptococcus gordonii, increasing the fermentation of complex carbohydrates and lactic-acid production ${ }^{49}$. Lactic acid is the preferred carbon source for V. atypica. $\mathbf{b}$ | LuxS-dependent autoinducer-2 (Al-2) production is important for biofilm development in Streptococcus gordonii. luxS mutants have altered biofilm formation and carbohydrate metabolism ${ }^{93,94}$. $\mathrm{Al}-2$ is also produced by several oral bacteria. c|LuxS activity has been shown to be important for S. gordonii to form mixed biofilms with Porphyromonas gingivalis ${ }^{94} . \mathbf{d}$ | The natural competence of Streptococcus mutans is enhanced in biofilms through the oligopeptide competencestimulating peptide (CSP) signalling pathway ${ }^{95}$. CSP signalling also contributes to biofilm formation in $\mathrm{S}$. mutans $^{96}$. In addition to chemical signalling between different species, there are also many specific physical interactions that contribute to the temporal and spatial organization of these communities ${ }^{3,47,48}$.

Niche partitioning

The division of a limiting resource(s) among the species that share it.

Flushing The removal of chemicals and/or individuals from an area by a current or flow through the area. a recent phenomenon, and the AHL molecules that are produced by different isolates of both organisms vary ${ }^{67,68}$, possibly leading to complex interactions between different isolates with overlapping activating and inhibitory signals. This would be similar to the diversity and grouping in oligopeptide signals that are observed in S. aureus ${ }^{24}$.

Our lack of understanding of the relationship between species is also illustrated in the case of the complex communities that form in the human oral cavity. Although it is well known that these communities comprise ordered spatial arrangements ${ }^{3,47,48}$ (FIG. 4), the exact nature of the relationship between species remains unclear. Therefore, the interaction between S. gordonii and V. atypica ${ }^{49}$, discussed previously, represents an example of an interaction that has yet to be clearly resolved with respect to communication, cues or chemical manipulation.

The intricate organization and interaction of bacteria within dental plaques is not unique, but represents one of the better-characterized complex microbial communities. In fact, most microorganisms probably exist in similar complex communities that exhibit temporal and spatial organization. The extent to which these communities are determined by chemical interactions, such as signalling and chemical warfare, as well as competition, niche partitioning and environmental limitations (flushing, temporal and spatial heterogeneity) is an area ripe for investigation.

It is also possible to have stable associations between species that are not dependent on chemical signalling or detection through alteration of the environment. An example is the association of yeast and bacteria that are commonly found on grapes. In the laboratory, this can be mimicked by growth in grape juice or in laboratory media with high concentrations of glucose. The yeast
Saccharomyces cerevisiae grows well in monoculture or in co-culture with the bacteria Pseudomonas putida ${ }^{69}$. In monoculture, $P$. putida can grow initially but does not survive because it ferments the carbohydrate, leading to the acidification of the environment, which it cannot tolerate. When grown together, however, both the bacteria and the yeast survive. This is the consequence of the yeast altering the environment by removing the high concentration of carbohydrate and reducing the acidification of the media by the bacteria ${ }^{69}$. In this case, it is not a response of the bacteria to a chemical produced by the yeast; it is a consequence of the yeast altering the environment that leads to the apparent interaction.

Interactions between bacteria and their host. In addition to bacteria-bacteria interactions, many bacteria are highly evolved for interaction with their eukaryotic hosts - in many instances, cell-cell-signalling systems of the bacteria have been implicated in the interaction. The best example is that of the symbiotic interaction of $V$. fischeri and its eukaryotic host, the bobtail squid ${ }^{16-18}$. Bacterial chemotaxis towards compounds found in the squid's light-organ mucus $^{70}$ and colonization of the light organ require motility $^{71,72}$. Quorum-sensing pathways control genes that are involved in motility, biofilm formation and colonization, in addition to the genes for light production ${ }^{73}$. Two other quorum-sensing systems, AinS and LuxS, in addition to the canonical LuxI/LuxR system, contribute to light production and colonization ${ }^{73,74}$. On the host side, it is known that colonization by the bacterium is necessary for normal development of the light organ ${ }^{75-77}$.

Perhaps most remarkably, the ability of the bacteria to produce light is important for them to remain competitive in the light organ. Mutants that are defective in the regulation of the lux genes or in the luciferase itself are less competitive in the colonization and long-term survival in the light organ than bacteria that are competent in light production ${ }^{78}$. It is not clear how the host can select specifically for luminescence-competent bacteria. It has been suggested that in the light organ, luciferase activity might be used to reduce the level of free oxygen $\left(\mathrm{O}_{2}\right.$ is consumed in the light-producing reaction), consequently reducing toxic reactive-oxygen species that are generated by the host ${ }^{78,79}$.

Evidence that a host might have the means to prevent cheating by symbiotic bacteria also comes from work on rhizobia and their legume host. In an elegant study, Kiers et al. ${ }^{80}$ showed that soybeans could penalize rhizobia that fail to fix nitrogen inside their root nodules. This apparently happened by plants decreasing oxygen supply to cheating, non-nitrogen-fixing rhizobia. By sanctioning cheater rhizobia, the plant effectively prevents their spread and therefore stabilizes the mutualistic symbiosis with the bacteria.

Most multicellular organisms are colonized to varying degrees by commensal flora. In humans, bacteria outnumber host cells by at least an order of magnitude, and the oral cavity and the intestinal tract are home to complex communities of microorganisms. The role of interspecies signalling among bacteria and between bacteria and their host is only beginning to be investigated; 
there is a vast array of interactions between commensal flora and the host immune system that are only partially understood. Undoubtedly, bacterially derived small molecules will be an important part of this dynamic ecology, and specific roles of quorum-sensing signals are being defined in some systems.

Some interactions of bacteria with their eukaryotic hosts provide examples that might best be described as chemical manipulation. One such example is the action of AHLs on the immune system. It has been shown that the $N$-3-oxododecanoyl homoserine lactone that is produced by $P$. aeruginosa can have varying effects on host cells, including the induction of apoptosis ${ }^{81,82}$ and immunomodulatory activity ${ }^{83-86}$. Therefore, it seems that $P$. aeruginosa is capable of manipulating its host through these small quorum-sensing chemicals. Interestingly, host cells have the capability to inactivate the AHL system $^{87}$, implying chemical warfare between the host and the pathogen. Another example is the production of quorum-sensing inhibitory molecules by the host, as seen in the marine algae Delisea pulchra, which produces a brominated furanone that acts as a competitive inhibitor of the AHL-signalling system of bacteria that colonize the surface of the algae $\mathrm{e}^{88,89}$.

\section{Perspectives}

This review reveals that the nature of interactions through quorum-sensing chemicals is not simply cooperative communication, but involves other interactions such as cues and chemical manipulations. Furthermore, these chemical signals might also have a role in conflicts, both within and between species. The nature of the chemical interaction is important to take into account when studying why and how bacteria react to the chemical substances produced by other bacteria or the host, and the molecular basis of autoinducers (speed of evolution, quantity of substances produced, specificity of the chemical, and so on). It should be emphasized that whether the chemical moiety is defined as a signal or a cue does not alter the significance of the interaction. Moreover, attempting to define the interaction in these terms, rather than with more generic descriptors, might provide insights into the structure of the particular microbial community. In broader terms, it is also important to bridge the link between microbiology and the field of ecology-evolution to make progress in our understanding of quorum sensing, and to develop bacteria as model organisms in ecology-evolution.
Greenberg, E. P. Bacterial communication: tiny teamwork. Nature 424, 134 (2003).

Henke, J. M. \& Bassler, B. L. Bacterial social engagements. Trends Cell Biol. 14, 648-656 (2004).

3. Kolenbrander, P. E., Egland, P. G., Diaz, P. I. \& Palmer, R. J. Jr. Genome-genome interactions: bacterial communities in initial dental plaque. Trends Microbiol. 13, 11-15 (2005)

4. Lyon, G. J. \& Novick, R. P. Peptide signaling in Staphylococcus aureus and other Gram-positive bacteria. Peptides 25, 1389-1403 (2004).

5. Parsek, M. R. \& Greenberg, E. P. Sociomicrobiology: the connections between quorum sensing and biofilms. Trends Microbiol. 13, 27-33 (2005).

6. Crespi, B. J. The evolution of social behavior in microorganisms. Trends Ecol. Evol. 16, 178-183 (2001).

7. Bourke, A. F. G. \& Franks, N. R. Social Evolution in Ants (Princeton University Press, Princeton, 1995).

. Hamilton, W. D. The genetical evolution of social behaviour. I. J. Theor. Biol. 7, 1-16 (1964).

9. Hamilton, W. D. The genetical evolution of social behaviour. II. J. Theor. Biol. 7, 17-52 (1964)

10. Keller, L. Levels of Selection in Evolution (Princeton University Press, Princeton 1999).

A book that reviews examples of conflicts that occur at the different levels of biological organization (for example, between genes within organisms and between organisms within social groups).

11. Szathmary, E. \& Maynard Smith, J. The Major Transitions in Evolution (Freeman Press, Oxford 1995).

12. Keller, L. \& Reeve, H. K. Kin selection. In Encyclopedia of Evolution 595-600 (Oxford University Press, Oxford, 2002).

13. Queller, D. C. Does population viscosity promote kin selection? Trends Ecol. Evol. 7, 322-324 (1992).

14. Redfield, R. J. Is quorum sensing a side effect of diffusion sensing? Trends Microbiol. 10, 365-370 (2002).

A thought-provoking commentary that challenges the conventional view of quorum sensing.

15. Shompole, S. et al. Biphasic intracellular expression of Staphylococcus aureus virulence factors and evidence for Agr-mediated diffusion sensing. Mol. Microbiol. 49, 919-927 (2003)

16. McFall-Ngai, M. J. Negotiations between animals and bacteria: the 'diplomacy' of the squid-Vibrio symbiosis. Comp. Biochem. Physiol. A Mol. Integr. Physiol. 126, 471-480 (2000).

17. Ruby, E. G. The Euprymna scolopes-Vibrio fischeri symbiosis: a biomedical model for the study of bacterial colonization of animal tissue. J. Mol. Microbiol. Biotechnol. 1, 13-21 (1999).
18. Visick, K. L. Layers of signaling in a bacterium-host association. J. Bacteriol. 187, 3603-3606 (2005).

19. Hastings, J. W. \& Greenberg, E. P. Quorum sensing: the explanation of a curious phenomenon reveals a common characteristic of bacteria. J. Bacteriol. 181 2667-2668 (1999)

20. Sachs, J. L., Mueller, U. G., Wilcox, T. P. \& Bull, J. J. The evolution of cooperation. Q. Rev. Biol. 79, 135-160 (2004)

A review of the mechanisms and selective forces that can maintain cooperation within and between species.

21. Manefield, M. \& Turner, S. L. Quorum sensing in context: out of molecular biology and into microbial ecology. Microbiology 148, 3762-3764 (2002).

22. Kleerebezem, M., Quadri, L. E., Kuipers, O. P. \& de Vos, W. M. Quorum sensing by peptide pheromones and two-component signal-transduction systems in Gram-positive bacteria. Mol. Microbiol. 24 895-904 (1997).

23. Mayville, P. et al. Structure-activity analysis of synthetic autoinducing thiolactone peptides from Staphylococcus aureus responsible for virulence. Proc. Natl Acad. Sci. USA 96, 1218-1223 (1999).

24. Ji, G., Beavis, R. \& Novick, R. P. Bacterial interference caused by autoinducing peptide variants. Science 276, 2027-2030 (1997).

25. Lyon, G. J., Wright, J. S., Muir, T. W. \& Novick, R. P. Key determinants of receptor activation in the agr autoinducing peptides of Staphylococcus aureus. Biochemistry 41, 10095-10104 (2002).

26. Eberl, L. $N$-acyl homoserinelactone-mediated gene regulation in Gram-negative bacteria. Syst. Appl. Microbiol. 22, 493-506 (1999).

27. Fuqua, C., Parsek, M. R. \& Greenberg, E. P. Regulation of gene expression by cell-to-cell communication: acyl-homoserine lactone quorum sensing. Annu. Rev. Genet. 35, 439-468 (2001).

28. Pearson, J. P. et al. Structure of the autoinducer required for expression of Pseudomonas aeruginosa virulence genes. Proc. Natl Acad. Sci. USA 91 197-201 (1994).

29. Winson, M. K. et al. Multiple $N$-acyl--Lhomoserine lactone signal molecules regulate production of virulence determinants and secondary metabolites in Pseudomonas aeruginosa. Proc. Natl Acad. Sci. USA 92, 9427-9431 (1995)

30. Shaw, P. D. et al. Detecting and characterizing $N$-acylhomoserine lactone signal molecules by thin-layer chromatography. Proc. Natl Acad. Sci. USA 94, 6036-6041 (1997)
31. Surette, M. G., Miller, M. B. ¿ Bassler, B. L. Quorum sensing in Escherichia coli, Salmonella typhimurium, and Vibrio harveyi: a new family of genes responsible for autoinducer production. Proc Natl Acad. Sci. USA 96, 1639-1644 (1999).

32. Xavier, K. B. \& Bassler, B. L. LuxS quorum sensing: more than just a numbers game. Curr. Opin. Microbiol. 6, 191-197 (2003)

33. Vendeville, A., Winzer, K., Heurlier, K., Tang, C. M. \& Hardie, K. R. Making 'sense' of metabolism: autoinducer-2, LuxS and pathogenic bacteria. Nature Rev. Microbiol. 3, 383-396 (2005)

34. Sun, J., Daniel, R., Wagner-Dobler, I. \& Zeng, A. P. Is autoinducer-2 a universal signal for interspecies communication: a comparative genomic and phylogenetic analysis of the synthesis and signal transduction pathways. BMC Evol. Biol. 4, 36 (2004).

35. Schauder, S., Shokat, K., Surette, M. G. \& Bassler, B. L. The LuxS family of bacterial autoinducers: biosynthesis of a novel quorum-sensing signal molecule. Mol. Microbiol. 41, 463-476 (2001).

36. Burgess, N. A. et al. LuxS-dependent quorum sensing in Porphyromonas gingivalis modulates protease and haemagglutinin activities but is not essential for virulence. Microbiology 148, 763-772 (2002)

37. Chen, X. et al. Structural identification of a bacterial quorum-sensing signal containing boron. Nature $\mathbf{4 1 5}$ 545-549 (2002)

This paper reports the structure of AI-2 as it is bound in the Vibrio harveyi receptor LuxP complexed with boron (see also reference 41).

38. Miller, S. T. et al. Salmonella typhimurium recognizes a chemically distinct form of the bacterial quorumsensing signal Al-2. Mol. Cell 15, 677-687 (2004).

39. Winzer, K., Hardie, K. R. \& Williams, P. LuxS and autoinducer-2: their contribution to quorum sensing and metabolism in bacteria. Adv. Appl. Microbiol. 53 , 291-396 (2003)

40. Travisano, M. \& Velicer, G. J. Strategies of microbial cheater control. Trends Microbiol. 12, 72-78 (2004).

41. Velicer, G. J. Social strife in the microbial world. Trends Microbiol. 11, 330-337 (2003).

This review provides examples of selfish strategies that undermine cooperative behaviour across various microbial systems.

42. Maynard Smith, J. \& Harper, D. Animal Signals (Oxford University Press, Oxford, 2003). A book that discusses, from a theoretical perspective, the problem of communication in animals.

43. Duan, K., Dammel, C., Stein, J., Rabin, H. \& Surette, M. G. Modulation of Pseudomonas aeruginosa gene expression by host microflora through interspecies communication. Mol. Microbiol. 50, 1477-1491 (2003). 
Demonstrates the interaction of oropharyngeal flora with $P$. aeruginosa, which results in enhanced pathogenicity. This is an example of the pathogen using $\mathrm{Al}-2$ that is produced by other bacteria as a cue.

44. Varaldi, J. et al. Infectious behavior in a parasitoid Science 302, 1930 (2003).

45. Thomas, F., Adamo, S. \& Moore, J. Parasitic manipulation: where are we and where should we go? Behav. Processes 68, 185-199 (2005).

46. Werren, J. H. Biology of Wolbachia. Annu. Rev. Entomol. 42, 587-609 (1997)

47. Kolenbrander, P. E. et al. Communication among oral bacteria. Microbiol. Mol. Biol. Rev. 66, 486-505 (2002).

48. Marsh, P. D. Dental plaque as a microbial biofilm Caries Res. 38, 204-211 (2004).

49. Egland, P. G., Palmer, R. J. Jr \& Kolenbrander, P. E. Interspecies communication in Streptococcus gordonii-Veillonella atypica biofilms: signaling in flow conditions requires juxtaposition. Proc. Natl Acad. Sci. USA 101, 16917-16922 (2004). Demonstrates an example of chemical manipulation between two oral bacteria, in which one strain produces a signal that alters the metabolism of the other. It also demonstrates that signalling occurs only on a very local scale.

50. Griffin, A. S., West, S. A. \& Buckling, A. Cooperation and competition in pathogenic bacteria. Nature $\mathbf{4 3 0}$ 1024-1027 (2004).

Shows that experimental manipulation of relatedness affects the level cooperative siderophore production in $P$. aeruginosa.

51. Fiegna, F. \& Velicer, G. J. Exploitative and hierarchical antagonism in a cooperative bacteria. PLoS Biol. 3 , 1980-1087 (2005)

52. Escalante, R. \& Vicente, J. J. Dictyostelium discoideum: a model system for differentiation and patterning. Int. J. Dev. Biol. 44, 819-835 (2000).

53. Weijer, C. J. Dictyostelium morphogenesis. Curr. Opin. Genet. Dev. 14, 392-398 (2004).

54. Kaiser, D. Signaling in myxobacteria. Annu. Rev. Microbiol. 58, 75-98 (2004).

55. Kaplan, H. B. Multicellular development and gliding motility in Myxococcus xanthus. Curr. Opin. Microbiol. 6, 572-577 (2003).

56. Sogaard-Andersen, L. Cell polarity, intercellular signalling and morphogenetic cell movements in Myxococcus xanthus. Curr. Opin. Microbiol. 7 587-593 (2004)

57. Shimkets, L. J. Intercellular signaling during fruitingbody development of Myxococcus xanthus. Annu. Rev. Microbiol. 53, 525-549 (1999)

58. Jelsbak, L. \& Sogaard-Andersen, L. The cell surfaceassociated intercellular C-signal induces behavioral changes in individual Myxococcus xanthus cells during fruiting body morphogenesis. Proc. Natl Acad. Sci. USA 96, 5031-5036 (1999)

59. Kruse, T., Lobedanz, S., Berthelsen, N. M. \& SogaardAndersen, L. C-signal: a cell surface-associated morphogen that induces and co-ordinates multicellular fruiting body morphogenesis and sporulation in Myxococcus xanthus. Mol. Microbiol. 40, 156-168 (2001).

60 Strassmann, J. E. Bacterial cheaters. Nature 404 555-556 (2000)

61. Velicer, G. J., Kroos, L. \& Lenski, R. E. Developmental cheating in the social bacterium Myxococcus xanthus. Nature 404, 598-601 (2000)

Demonstrates that some mutants that are defective for fruiting-body development are overrepresented among the spores that are produced. This shows a trade-off between cooperative traits that is beneficial to the group but costly to the individual.

62. Chandler, J. R. \& Dunny, G. M. Enterococcal peptide sex pheromones: synthesis and control of biological activity. Peptides 25, 1377-1388 (2004).

63. Dunny, G. M., Antiporta, M. H. \& Hirt, H. Peptide pheromone-induced transfer of plasmid pCF10 in Enterococcus faecalis: probing the genetic and molecular basis for specificity of the pheromone response. Peptides 22, 1529-1539 (2001).

64. Diaz, E. Bacterial degradation of aromatic pollutants: a paradigm of metabolic versatility. Int. Microbiol. 7 , 173-180 (2004).
65. Lewenza, S., Visser, M. B. \& Sokol, P. A. Interspecies communication between Burkholderia cepacia and Pseudomonas aeruginosa. Can. J. Microbiol. 48, 707-716 (2002).

66. Riedel, K. et al. N-acylhomoserine-lactone-mediated communication between Pseudomonas aeruginosa and Burkholderia cepacia in mixed biofilms. Microbiology 147, 3249-3262 (2001).

67. Chambers, C. E., Visser, M. B., Schwab, U. \& Sokol, P. A. Identification of $\mathrm{N}$-acylhomoserine lactones in mucopurulent respiratory secretions from cystic fibrosis patients. FEMS Microbiol. Lett. 244. 297-304 (2005).

68. Geisenberger, O. et al. Production of $\mathrm{N}$-acyl-Lhomoserine lactones by $P$. aeruginosa isolates from chronic lung infections associated with cystic fibrosis. FEMS Microbiol Lett 184, 273-278 (2000).

69. Romano, J. D. \& Kolter, R. PseudomonasSaccharomyces interactions: influence of fungal metabolism on bacterial physiology and survival. $J$. Bacteriol. 187, 940-948 (2005).

70. DeLoney-Marino, C. R., Wolfe, A. J. \& Visick, K. L. Chemoattraction of Vibrio fischeri to serine, nucleosides, and $\mathrm{N}$-acetylneuraminic acid, a component of squid light-organ mucus. Appl. Environ Microbiol. 69, 7527-7530 (2003).

71. Graf, J., Dunlap, P. V. \& Ruby, E. G. Effect of transposon-induced motility mutations on colonization of the host light organ by Vibrio fischeri. J. Bacteriol. 176, 6986-6991 (1994).

72. Millikan, D. S. \& Ruby, E. G. Alterations in Vibrio fischeri motility correlate with a delay in symbiosis initiation and are associated with additional symbiotic colonization defects. Appl. Environ. Microbiol. 68, 2519-2528 (2002)

73. Lupp, C. \& Ruby, E. G. Vibrio fischeri uses two quorum-sensing systems for the regulation of early and late colonization factors. J. Bacteriol. 187 3620-3629 (2005)

74. Lupp, C. \& Ruby, E. G. Vibrio fischeri LuxS and AinS comparative study of two signal synthases. $J$. Bacteriol. 186, 3873-3881 (2004).

75. Doino, J. \& McFall-Ngai, M. transient exposure to symbiosis-competent bacteria induces light organ morphogenesis in the host squid. Biol. Bull. 189, 347-355 (1995).

76. Doino Lemus, J. \& McFall-Ngai, M. J. Alterations in the proteome of the Euprymna scolopes light organ in response to symbiotic Vibrio fischeri. Appl. Environ. Microbiol. 66, 4091-4097 (2000).

77. Foster, J. S., Apicella, M. A. \& McFall-Ngai, M. J. Vibrio fischeri lipopolysaccharide induces developmental apoptosis, but not complete morphogenesis, of the Euprymna scolopes symbiotic light organ. Dev. Biol. 226, 242-254 (2000)

78. Visick, K. L., Foster, J., Doino, J., McFall-Ngai, M. \& Ruby, E. G. Vibrio fischeri lux genes play an important role in colonization and development of the host light organ. J. Bacteriol 182, 4578-4586 (2000).

79. Ruby, E. G. \& McFall-Ngai, M. J. Oxygen-utilizing reactions and symbiotic colonization of the squid light organ by Vibrio fischeri. Trends Microbiol. 7 , 414-420 (1999).

80 Kiers, E. T., Rousseau, R. A., West, S. A \& Denison, R. F. Host sanctions and the legumeRhizobium mutualism. Nature 425, 78-81 (2003).

81. Li, L., Hooi, D., Chhabra, S. R., Pritchard, D. \& Shaw, P. E. Bacterial $N$-acylhomoserine lactoneinduced apoptosis in breast carcinoma cells correlated with down-modulation of STAT3. Oncogene 23 4894-4902 (2004)

82. Tateda, K. et al. The Pseudomonas aeruginosa autoinducer $\mathrm{N}$-3-oxododecanoyl homoserine lactone accelerates apoptosis in macrophages and neutrophils. Infect. Immun. 71, 5785-5793 (2003).

83. Ritchie, A. J. et al. The Pseudomonas aeruginosa quorum-sensing molecule $\mathrm{N}$-3-(oxododecanoyl)- $\mathrm{L}-$ homoserine lactone inhibits T-cell differentiation and cytokine production by a mechanism involving an early step in T-cell activation. Infect. Immun. $\mathbf{7 3}$ 1648-1655 (2005)

84. Smith, R. S., Kelly, R., Iglewski, B. H. \& Phipps, R. P. The Pseudomonas autoinducer $N$-(3-oxododecanoyl) homoserine lactone induces cyclooxygenase- 2 and prostaglandin E2 production in human lung fibroblasts: implications for inflammation. J. Immunol. 169, 2636-2642 (2002).
85. Telford, G. et al. The Pseudomonas aeruginosa quorum-sensing signal molecule $N$-(3-oxododecanoyl)-L-homoserine lactone has immunomodulatory activity. Infect. Immun. 66, 36-42 (1998)

86. Hooi, D. S., Bycroft, B. W., Chhabra, S. R., Williams, P. $\&$ Pritchard, D. I. Differential immune modulatory activity of Pseudomonas aeruginosa quorum-sensing signal molecules. Infect. Immun. 72, 6463-6470 (2004).

87. Chun, C. K., Ozer, E. A., Welsh, M. J., Zabner, J. \& Greenberg, E. P. Inactivation of a Pseudomonas aeruginosa quorum-sensing signal by human airway epithelia. Proc. Natl Acad. Sci. USA 101, 3587-3590 (2004).

88. Givskov, M. et al. Eukaryotic interference with homoserine lactone-mediated prokaryotic signalling J. Bacteriol. 178, 6618-6622 (1996).

89. Manefield, M. et al. Evidence that halogenated furanones from Delisea pulchra inhibit acylated homoserine lactone (AHL)-mediated gene expression by displacing the AHL signal from its receptor protein Microbiology 145, 283-291 (1999).

90. Solomon, J. M., Lazazzera, B. A. \& Grossman, A. D. Purification and characterization of an extracellular peptide factor that affects two different developmental pathways in Bacillus subtilis. Genes Dev. 10, 2014-2024 (1996).

91. Tummler, B. et al. Infections with Pseudomonas aeruginosa in patients with cystic fibrosis. Behring Inst. Mitt. 98, 249-255 (1997).

92. Schaber, J. A. et al. Analysis of quorum sensingdeficient clinical isolates of Pseudomonas aeruginosa. J. Med. Microbiol. 53, 841-853 (2004).

93. Blehert, D. S., Palmer, R. J. Jr, Xavier, J. B. Almeida, J. S. \& Kolenbrander, P. E. Autoinducer 2 production by Streptococcus gordonii DL1 and the biofilm phenotype of a luxS mutant are influenced by nutritional conditions. J. Bacteriol. 185, 4851-4860 (2003).

94. McNab, R et al. LuxS-based signaling in Streptococcus gordonii: autoinducer 2 controls carbohydrate metabolism and biofilm formation with Porphyromonas gingivalis. J. Bacteriol. 185, 274284 (2003).

95 Li, Y H. Lau, P. C., Lee, J. H., Ellen, R. P \& Cvitkovitch, D. G. Natural genetic transformation of Streptococcus mutans growing in biofilms. J. Bacteriol. 183, 897-908 (2001).

96. Li, Y. H. et al. A quorum-sensing signaling system essential for genetic competence in Streptococcus mutans is involved in biofilm formation. J. Bacteriol. 184, 2699-2708 (2002).

\section{Acknowledgements}

We thank B. Bassler, C. Davidson, P. Greenberg, G. Velicer, K. Visick, P. Williams and four reviewers for their useful com ments on the manuscript. The authors were supported by grants from the Swiss National Science Foundation (L.K.) and the Canadian Institutes of Health Research (M.G.S.)

\section{Competing interests statement}

The authors declare no competing financial interests.

\section{DATABASES}

The following terms in this article are linked online to: Entrez Genome Project: http://www.ncbi.nlm.nih.gov/ entrez/query.fcgi?db=genomepr Agrobacterium tumefaciens | Chromobacterium violaceum | Myxococcus xanthus $\mid$ Pseudomonas aeruginosa $\mid$ Pseudomonas putida | Saccharomyces cerevisiae | Salmonella enterica serovar Typhimurium | Staphylococcus aureus | Streptococcus gordonii| Vibrio fischeri

\section{FURTHER INFORMATION}

Laurent Keller's homepage:

http://www.unil.ch/dee/page6763 fr.html

Micheal G. Surette's homepage:

http://www.med.ucalgary.ca/webs/bprg/Surette/

Surette.html

Entrez PubMed: http://www.ncbi.nlm.nih.gov/entrez/ query.fcgi

Access to this links box is available online. 